University of Wollongong

Research Online

Faculty of Informatics - Papers (Archive)

Faculty of Engineering and Information

Sciences

18-10-2006

\title{
Adaptive low-complexity MMSE channel estimation for OFDM
}

\author{
Darryn Lowe \\ University of Wollongong, darrynl@uow.edu.au \\ Xiaojing Huang \\ University of Wollongong, huang@uow.edu.au
}

Follow this and additional works at: https://ro.uow.edu.au/infopapers

Part of the Physical Sciences and Mathematics Commons

\section{Recommended Citation \\ Lowe, Darryn and Huang, Xiaojing: Adaptive low-complexity MMSE channel estimation for OFDM 2006. https://ro.uow.edu.au/infopapers/406}

Research Online is the open access institutional repository for the University of Wollongong. For further information contact the UOW Library: research-pubs@uow.edu.au 


\title{
Adaptive low-complexity MMSE channel estimation for OFDM
}

\begin{abstract}
In this paper we present extremely low-complexity adaptive infinite impulse response (IIR) filters that approximate minimum mean square error (MMSE) channel estimation in orthogonal frequency-division multiplexing (OFDM) systems. We show how the packet error rate (PER) can be significantly improved over conventional zero-forcing (ZF) estimation without incurring a significant increase in computational complexity. All quantitative results are provided in the context of multi-band OFDM (MB-OFDM) ultrawideband (UWB) with standard IEEE channel models.
\end{abstract}

\section{Keywords}

UWB, ultra-wideband, MB-OFDM, equalization, estimation, MMSE

\section{Disciplines}

Physical Sciences and Mathematics

\section{Publication Details}

This paper was originally published as: Lowe, D \& Huang, X, Adaptive low-complexity MMSE channel estimation for OFDM, 2006 International Symposium on Communications and Information Technologies (ISCIT), Bangkok, Thailand, 18-20 October 2006. Conference information is available here. 


\title{
Adaptive Low-Complexity MMSE Channel Estimation for OFDM
}

\author{
Darryn Lowe and Xiaojing Huang \\ School of Electrical, Computer and Telecommunications Engineering \\ University of Wollongong \\ Wollongong, Australia, 2522 \\ Email: \{darrynl, huang\}@uow.edu.au
}

\begin{abstract}
In this paper we present extremely low-complexity adaptive infinite impulse response (IIR) filters that approximate minimum mean square error (MMSE) channel estimation in orthogonal frequency-division multiplexing (OFDM) systems. We show how the packet error rate (PER) can be significantly improved over conventional zero-forcing (ZF) estimation without incurring a significant increase in computational complexity. All quantitative results are provided in the context of multi-band OFDM (MB-OFDM) ultra-wideband (UWB) with standard IEEE channel models.
\end{abstract}

\section{INTRODUCTION}

Coherent signalling over frequency-selective channels requires that the receiver estimate and equalize the channel before demodulating the symbol constellations. One of the most significant advantages of orthogonal frequency division multiplexing (OFDM) systems [1] is that channel estimation and equalization are conceptually and computationally simple. This is one of many reasons why OFDM systems are becoming increasingly popular in applications ranging from wireless personal area networks (WPANs) to digital television.

The simplest means for OFDM channel estimation is a zero-forcing (ZF) approximation of $N$ complex coefficients to rotate and scale each of the symbol's $N$ subcarriers. To keep complexity low, many OFDM equalizers disregard the potentially significant correlation between subcarriers. This means that the magnitude of the additive white Gaussian noise (AWGN) that degrades the channel estimation is independent of the channel length. In other words, an impulsive flat-fading pure-AWGN channel needlessly suffers from the same channel estimation error as a highly frequency-selective channel. This means that the packet error rate (PER) is suboptimal in short channels.

To improve performance and make full use of subcarrier correlation, a minimum mean square error (MMSE) estimator can be used. Since a direct MMSE estimation requires an $N \times N$ matrix multiplication [2], it is prohibitively expensive in highrate low-power systems like multi-band OFDM (MB-OFDM) [3], which is the first ultra-wideband (UWB) technology to obtain international standardization [4]. Although the channel estimation techniques developed in this paper are applicable to almost any OFDM system, our results are presented in the context of MB-OFDM so that the performance improvements can be balanced against the increased complexity.
This paper investigates how to balance the quality and the complexity of OFDM channel estimation in the context of the MB-OFDM standard. We begin with a theoretical analysis in Section II, where we obtain an upper bound on performance. Section III then develops ultra-low complexity approximatelyMMSE estimation techniques. In Section IV, we exploit this complexity reduction to enable the estimation to be adaptive to instantaneous channel conditions. The final PER is then analyzed through Monte Carlo simulations in Section IV and the findings summarized in the conclusions of Section V.

\section{Motivation}

We model $^{1}$ an OFDM system as

$$
\mathbf{y}=\mathbf{X h}+\mathbf{n}
$$

where $\mathbf{y}$ is the post-FFT received vector, $\mathbf{X}$ is a diagonal matrix containing the transmitted symbol constellations, $\mathbf{h}$ is a complex channel attenuation vector and $\mathbf{n}$ is a vector of independent and identically distributed complex, zero-mean, Gaussian noise variables with variance $\sigma_{n}^{2}$. Note that (1) is entirely in the frequency-domain. Without loss of generality, we assume that the channel is normalized such that $E\left\{\left|h_{k}\right|^{2}\right\}=1$ and $E\left\{\left|X_{k, k}\right|^{2}\right\}=1$.

The receiver channel estimation is usually performed with the aid of a known training sequence. This allows a ZF channel estimation to be easily obtained as

$$
\hat{\mathbf{h}}_{\mathrm{ZF}}=\mathbf{X}^{-1} \mathbf{y}=\mathbf{h}+\tilde{\mathbf{n}}
$$

where $\tilde{\mathbf{n}}=\mathbf{X}^{-1} \mathbf{n}$. Given our earlier assumption that $E\left\{\left|X_{k, k}\right|^{2}\right\}=1$, the variance of the AWGN denoted by $\tilde{\mathbf{n}}$ will remain $\sigma_{n}^{2}$. It is apparent that this ZF estimation does not exploit the correlation between subcarriers and that the meansquared error (MSE) of the channel estimate will be $\frac{1}{\sigma_{n}^{2}}$.

To minimize the MSE, an optimal linear estimation [5] can be denoted as

$$
\hat{\mathbf{h}}=\mathbf{W} \hat{\mathbf{h}}_{\mathrm{ZF}}
$$

where

$$
\mathbf{W}=\mathbf{R}_{h h}\left(\mathbf{R}_{h h}+\sigma_{n}^{2} \mathbf{I}\right)^{-1}
$$

${ }^{1}$ Throughout this paper, the following matrix notation conventions are adopted: [. $]^{H}$ denotes the Hermitian transpose; $[.]^{-1}$ the matrix inverse and $[.]_{k, n}$ the element of the $k^{\text {th }}$ row and $n^{\text {th }}$ column. 


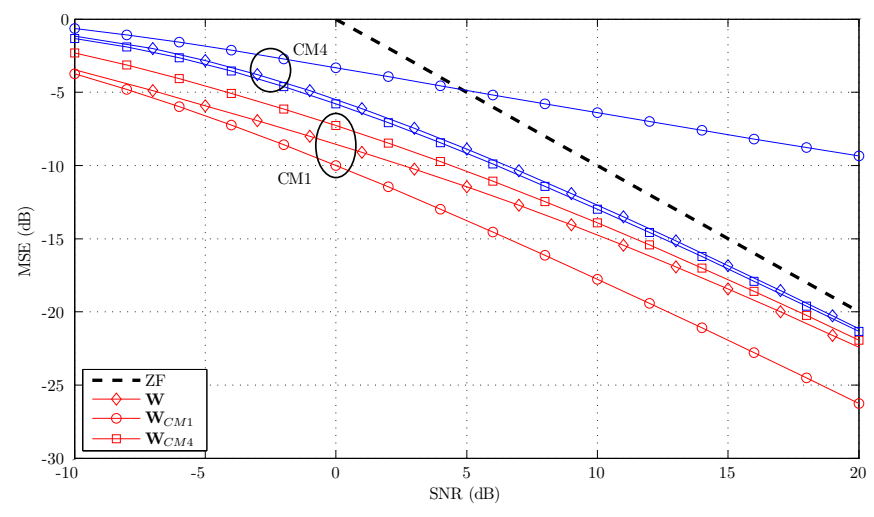

Fig. 1. MSE for different channel smoothing matrices $\mathbf{W}$.

with $\mathbf{R}_{h h}=E\left\{\mathbf{h h}^{H}\right\}$ denoting the auto-covariance matrix of the channel vector $\mathbf{h}$ and with $\mathbf{I}$ denoting the $N \times N$ identity matrix. Intuitively, (4) can be easily understood. When $\sigma_{n}^{2}=$ 0 and there is no AWGN, there is no need to exploit any subcarrier correlation and thus $\mathbf{W}=\mathbf{I}$. When the AWGN increases such that $\sigma_{n}^{2} \gg 1$, we obtain $\mathbf{W}=\frac{1}{\sigma_{n}^{2}} \mathbf{R}_{h h}$ and subcarrier correlation is fully leveraged to reduce the impact of the noise as much as possible.

Fig. 1 shows how critical it is that the channel autocovariance matrix $\mathbf{R}_{h h}$, used to calculate $\mathbf{W}$, be representative of channel conditions. The reference filter, denoted as $\mathbf{W}$, is obtained from both CM1 and CM4 channel impulse responses (CIRs). The channel-specific filters, denoted as $\mathbf{W}_{\mathrm{CM} 1}$ and $\mathbf{W}_{\mathrm{CM} 4}$, use CIRs from only their respective channel model. We observe that minimum MSE is achieved when the channel statistics closely match the actual CIR.

For example, if $\mathbf{W}$ is based on an overestimation of the channel length, as occurs when $\mathbf{W}_{\mathrm{CM} 4}$ is used in CM1 channels, the correlation between subcarriers is under-utilized and the MSE increases by several $\mathrm{dB}$ relative to the reference $\mathbf{W}$. Despite this, the performance is still superior than that of the original ZF channel estimate which allows us to conclude that even such sub-optimal exploitation of subcarrier correlation is often better than none at all.

The losses that arise when $\mathbf{W}$ underestimates the channel length, as is the case when $\mathbf{W}_{\mathrm{CM} 1}$ is used in CM4 channels, are much more serious. Under these circumstances, it is possible for the filtering to degrade the ZF channel estimate. This is because independent subcarriers are erroneously correlated.

We conclude that MMSE channel estimation is only suitable when the statistical properties of the CIRs are well known. In the case of MB-OFDM, where the CIRs can vary significantly depending on the distance between of transmitter and receiver, it may even be necessary to adapt $\mathbf{W}$ in real-time. For example, if an UWB transceiver pair are placed within a few centimeters of each other, then all CIRs will tend to be from the short CM1 channel. If a $\mathbf{W}$ derived from a mix of all channel models were used, the subcarrier correlation would be underestimated and the MSE would be suboptimal.

Even when the statistics underlying $\mathbf{W}$ are known accu- rately, there are two additional challenges that complicate practical implementation of MMSE channel estimation. First, the filtering of the ZF estimate is computationally expensive. For example, direct implementation of (4) requires an expensive $N \times N$ matrix multiplication. Second, the IEEE channel models that underly Fig. 1 are quite broad. This means that although the average delay spread of CM4 is greater than that of CM1, individual realizations of the CIR will vary considerably. The CM1/CM4 classification is therefore not appropriate for a practical receiver.

In the following two sections, we present several solutions to these problems. First, in Section III, we show how the complexity can be reduced sufficient for implementation under the severe constraints imposed on MB-OFDM hardware. Then, in Section IV, we develop an adaptive algorithm that reuses calculations performed during packet synchronization.

\section{COMPLEXITY REDUCTION}

Consider the complex baseband CIR $\mathbf{g}=\mathbf{F}^{H} \mathbf{h}$ where $\mathbf{F}$ is the $N \times N$ FFT matrix with $[\mathbf{F}]_{k, n}=\frac{1}{\sqrt{N}} e^{-j 2 \pi k n / N}$. We can denote the time-domain channel auto-covariance matrix as

$$
\mathbf{R}_{g g}=\mathrm{E}\left[\mathbf{g g}^{H}\right]=\mathbf{F}^{H} \mathbf{R}_{h h} \mathbf{F}
$$

If we assume that each tap of $\mathrm{g}$ has a uniformly distributed phase between 0 and $2 \pi$, as is the case in the IEEE UWB channel models, then $\mathbf{R}_{g g}$ will be a diagonal matrix. This forces both $\mathbf{R}_{h h}$ and $\mathbf{W}$ to be circulant [6].

We can therefore denote MMSE smoothing as the circular convolution of a ZF channel estimate with an $N$-tap finite impulse response (FIR) filter. We express the impulse response of this filter, equivalent to the first row of $\mathbf{W}$, as $\mathbf{w}=$ $\left[w_{0}, w_{1}, \ldots, w_{N-1}\right]$. Note that our use of the term 'impulse response' is with regard to the filter $\mathbf{w}$; the fact that the filter is applied to a frequency-domain channel estimate is irrelevant.

Direct filtering of the $\mathrm{ZF}$ channel estimate is not computationally feasible since the a circular convolution would require $N^{2}$ complex multiplications. One way to reduce this complexity is to use fast convolution [2]. Unfortunately, this approach involves two FFT/IFFTs: one to transform the initial frequency-domain ZF estimate into the time-domain and one to transform the smoothed MMSE estimate back into the frequency-domain. Given that each FFT/IFFT incurs $\frac{N}{2} \log _{2} N$ complex multiplications, the total complexity of a fast-convolution approach would be $\mathcal{O}\left(N \log _{2} N+N\right)$. Although this is a significant improvement over the $\mathcal{O}\left(N^{2}\right)$ complexity needed for direct circular convolution, $1024 \mathrm{com}$ plex multiplications is still far too expensive for an MB-OFDM system where $N=128$.

Fast-convolution can be simplified by truncating the timedomain ZF estimate of the CIR to $M$ taps. Although this avoids $N-M$ complex multiplications, the continuing presence of an FFT/IFFT pair results in a still-too-high complexity of $\mathcal{O}\left(N \log _{2} N+1\right)$.

In the same way that we can truncate the filter to $M$ taps when using fast convolution, we can also truncate $w_{n}$ to $P$ taps when using direct circular convolution. Indeed, when 
$P^{2}<N \log _{2} N$, that the filter $\mathbf{w}$ will be so short that it would be more expensive to perform the FFT and IFFT needed for fast convolution. In the context of MB-OFDM, this means that direct circular convolution is to be preferred over fast convolution if the filter $\mathbf{w}$ is truncated to $P<30$ taps.

\section{A. Low-Complexity Filter Design}

To further reduce the often prohibitive complexity of MMSE channel estimation, we now develop several low complexity alternatives. To begin, we quantify the MSE of a channel estimate as

$$
\operatorname{MSE}=\frac{1}{N} \operatorname{Trace}\left(\mathbf{R}_{e e}\right)
$$

where $\mathbf{R}_{e e}$ is the auto-covariance matrix of the channel estimation error and is defined as

$$
\begin{aligned}
\mathbf{R}_{e e} & =E\left\{(\hat{\mathbf{h}}-\mathbf{h})(\hat{\mathbf{h}}-\mathbf{h})^{H}\right\} \\
& =\mathbf{W}\left(\mathbf{R}_{h h}+\sigma_{n}^{2} \mathbf{I}\right) \mathbf{W}^{H}-\mathbf{R}_{h h} \mathbf{W}^{H}-\mathbf{W R}_{h h}^{H}+\mathbf{R}_{h h}
\end{aligned}
$$

where $\mathbf{W}$ denotes the smoothing matrix. If $\mathbf{W}=\mathbf{I}$, then the smoothing filter is unused and $\mathrm{MSE}=\sigma_{n}^{2}$ since the $\mathrm{ZF}$ channel estimation is used as-is. We observe that defining $\mathbf{W}$ as per (4) yields the optimal MMSE[5].

We now present several low-complexity IIR filters that approximate $\mathbf{W}$. This is done by minimizing (7) with the filter coefficients as the unknowns. Since $\mathbf{R}_{h h}$ is only obtainable via numeric methods, the minimization must be performed as an iterative search. Although this is computationally intensive, the problem remains tractable since all the candidate filters are very low order. For example, the most complex filter that we consider has only three independent variables. Although any non-linear search algorithm could be used, we selected the simplex method [7] given its efficiency for low-dimension search spaces.

For each filter, we provide a transfer function and an impulse response. The impulse responses are expressed in the form $\mathbf{a}=\left[a_{0}, a_{1}, \ldots, a_{N-1}\right]$ and are incorporated into (7) through the circulant matrix $\hat{\mathbf{W}}_{a}$ which has a as its first row.

1) First-order IIR: The simplest approach to channel estimation smoothing is to use a first-order infinite impulse response (IIR) filter with real coefficients. The transfer function of this filter is

$$
\mathrm{TF}_{a}(z)=B \frac{z}{z-A}
$$

where $A$ is the coefficient controlling the rate of decay and $B$ is the gain. This filter's impulse response is

$$
a_{n}=B A^{n}
$$

with both $A$ and $B$ constrained to positive real numbers since complex coefficients introduce unwanted phase rotations in the filtered output. For stability, we also constrain $A<1$.
2) Second-order IIR: We also consider a second-order IIR filter with the transfer function

$$
\mathrm{TF}_{b}(z)=B\left(\frac{z}{z-A}+\frac{z}{z-C}\right)
$$

where $C$ is an additional real coefficient constrained to $C<1$ for stability. We denote the corresponding impulse response as

$$
b_{n}=B\left(A^{n}+C^{n}\right)
$$

3) Symmetric IIR: The correlation between OFDM subcarriers is symmetric. It is therefore desirable to consider both higher and lower subcarriers when smoothing the ZF channel estimation. Since the first- and second-order IIR filters defined thus far are not symmetric, they exploit only half of the available correlation. We solve this problem by defining a symmetric variant of the first-order filter of (9) as

$$
a_{n}^{\leftrightarrow}=a_{n}+a_{N-n}
$$

and similarly for $b_{n}^{\leftrightarrow}$.

In terms of hardware realization, a symmetric IIR filter can be easily implemented by adding the results from two independent IIR filters that each operating over the same input data in opposite directions.

4) Product Power Play: The most costly part of digital filtering is multiplication. If we are flexible with our filter coefficients, we can avoid multiplication by using a product power play (PPP) [8] to approximate each filter tap with the sum-and-difference of $Q$ binary shifts. In other words, $\alpha \approx \sum \pm 2^{\alpha_{1}} \pm 2^{\alpha_{2}} \pm \ldots \pm 2^{\alpha_{Q}}$ where $\alpha$ is a real-valued constant and $\alpha_{1}$ through $\alpha_{Q}$ are integers. Another benefit of this approach is that the integer constants $\alpha_{q}$ can be stored using very little memory. For example, in a receiver where the channel estimates are stored with 8-bits of precision, only $\log _{2} 8=3$ bits are required for each variable shift $\alpha_{q}$ if $0<\alpha \leq 1$. This memory consumption can be reduced even further if some $\alpha_{q}$ are fixed, as could be the case for coefficients with a small dynamic range.

We denote the impulse response of a PPP first-order IIR filter as $\mathbf{b}_{Q=2}^{\leftrightarrow}$, which denotes a $\mathbf{b} \leftrightarrow$ filter wherein each coefficient is approximated as the sum of two variable shifts. Note that it is also possible to use a PPP to simplify an FIR filter W. Unfortunately, the large number of taps in the FIR filter means that this will lead to large high-latency adder-trees.

\section{B. Performance Comparison}

Fig. 2 shows a comparison between the frequency responses

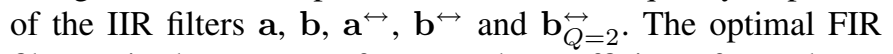
filter $\mathbf{w}$ is shown as a reference. The coefficients for each IIR filter were obtained by performing a simplex search on (6) to find the minimal error. In this example, $\mathbf{R}_{h h}$ was calculated using CM1 only and the SNR was arbitrarily set to $10 \mathrm{~dB}$. We observe that the most obvious difference between the optimal filter and the IIR approximations is that the optimal filter has an asymmetric frequency response. Conversely, as the IIR filters are constrained to purely real coefficients, their frequency responses are symmetric. In the context of MMSE 


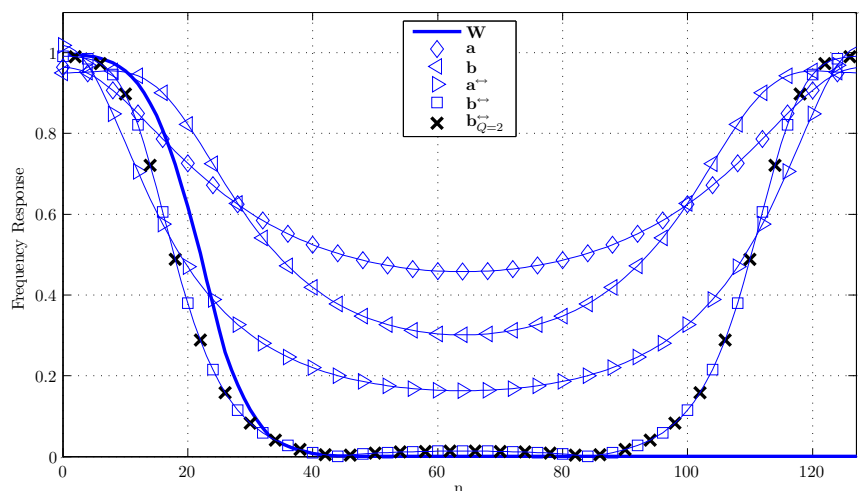

Fig. 2. Comparison of the frequency response of the approximate smoothing filters.

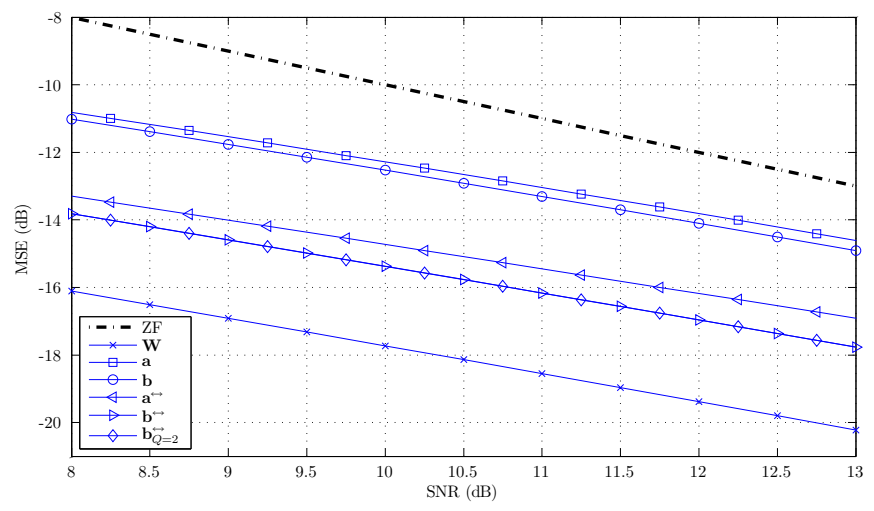

Fig. 3. MSE for different MMSE IIR filters.

channel estimation, this means that an IIR filter is not able to zero the tail of the ZF estimate of the CIR. Of the candidate IIR filters, we note that the best match to the optimal response is obtained by the symmetric second-order filter $\mathbf{b} \leftrightarrow$. We observe that the incremental loss incurred with a $Q=2$ PPP is trivial.

Fig. 3 shows the MSE of the same filters in a CM1 environment. The MSE of the original ZF estimate remains the inverse of the SNR and is consistent with the theoretical results of Fig. 1. We observe that the symmetric IIR filters perform significantly better than their asymmetric counterparts. As in Fig. 2, the losses due to a $Q=2$ quantization are trivial.

We conclude that $\mathbf{a} \mathbf{b}_{Q=2}^{\leftrightarrow}$ IIR filter is an effective approximation of optimal MMSE channel estimation.

\section{Complexity Comparison}

Table III-C summarizes the complexity of the candidate filters. The low-order IIR filters are much simpler than both implementations of the FIR filter w. For example, in an MBOFDM system where $N=128$, b incurs only 384 real multiplications as opposed to more than 1,000 complex multiplications for FIR fast-convolution. Although symmetrical IIR filters are double the complexity of their asymmetric variants, the fact that a PPP removes all multiplications makes the practical difference relatively minor. Therefore, although $\mathbf{a}_{Q=2}^{\leftrightarrow}$ has the lowest cost, the filter we use for further analysis is

\begin{tabular}{|c|l|l|}
\hline Filter & Implementation Notes & Complexity \\
\hline $\mathbf{w}$ & Direct Convolution & $N^{2}$ Complex Mults. \\
$\mathbf{w}$ & Fast Convolution & $N(1+\log N)$ Complex Mults. \\
\hline $\mathbf{a}$ & 1st-Order IIR & $2 N$ Real Mults. \\
$\mathbf{b}$ & 2nd-Order IIR & $3 N$ Real Mults. \\
\hline $\mathbf{a} \leftrightarrow$ & Symmetric 1st-order IIR & $4 N$ Real Mults. \\
$\mathbf{b} \leftrightarrow$ & Symmetric 2nd-order IIR & $6 N$ Real Mults. \\
\hline $\mathbf{a}_{Q}^{\leftrightarrow}=2$ & Product Power Play & $24 N$ Real Add/Subs. \\
$\mathbf{b}_{\leftrightarrow}^{\leftrightarrow}=2$ & Product Power Play & $34 N$ Real Add/Subs. \\
$\mathbf{b}_{Q}^{\leftrightarrow}=3$ & Product Power Play & $46 N$ Real Add/Subs. \\
\hline
\end{tabular}

TABLE I

COMPARISON OF IMPLEMENTATION COMPLEXITY OF FILTERING OPTIONS

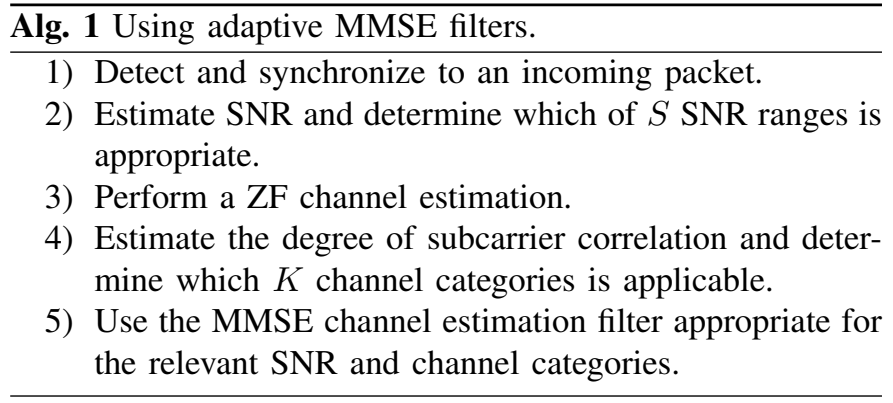

$\mathbf{b}_{Q=2}^{\leftrightarrow}$ given that 10 extra additions per subcarrier is not seen as prohibitively expensive.

\section{ADAptive Filters}

In previous work [9], it was recommended that an allpurpose $\mathbf{W}$ be calculated using a channel auto-covariance matrix $\mathbf{R}_{h h}$ that is representative of all possible channel conditions. The SNR used to derive this generic $\mathbf{W}$ should be relatively high given that a low SNR will lead to excessive correlation between subcarriers and thereby increase the MSE for short CIRs. This was seen in Fig. 1 when a $\mathbf{W}$ derived under CM1 was used in CM4.

In this paper, we have constructed several low complexity IIR approximations to the optimal MMSE filter. The preferred $\mathbf{b}_{Q=2}^{\leftrightarrow}$ filter is fully defined by the six constants $\left\{A_{1}, A_{2}, B_{1}, B_{2}, C_{1}, C_{2}\right\}$ that denote the PPP coefficients for $(A, B, C)$. This filter is very small as it requires only $6 \log _{2} B$ bits, with $B$ denoting the bits of precision in the $\mathrm{ZF}$ channel estimate, of read-only memory (ROM). For example, in an MB-OFDM receiver with an 8 bit ADC, the entire IIR filter can be stored in as little as 18 bits.

We can exploit the low ROM requirements to store several complimentary smoothing filters that are tuned for $S$ SNR ranges and $K$ classes of CIR. A receiver that use an adaptive MMSE channel estimation as per an algorithm similar to that of Alg. 1. This approach is only practical if steps 2 and 3 are low complexity.

Many receivers already estimate SNR during synchronization or as part of the ZF channel estimation. When a predefined training sequence is used, the SNR is trivially calculated as

$$
\mathrm{SNR}=\frac{\left|\sum t_{0}(n)+t_{1}(n)\right|^{2}}{\left|\sum t_{0}(n)-t_{1}(n)\right|^{2}}
$$




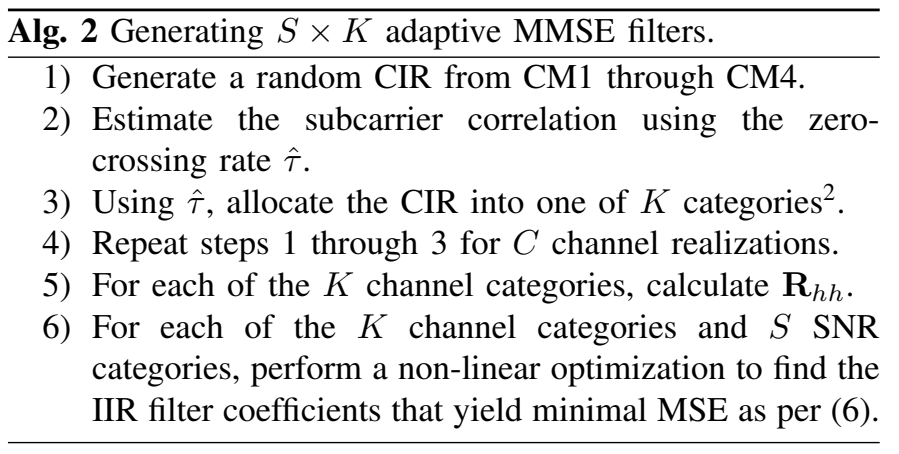

where $t_{0}(n)$ and $t_{1}(n)$ are the $n^{\text {th }}$ received samples of the first and second repetitions of the training sequence $t(n)$. Note that many standards, including MB-OFDM, require all receivers to estimate SNR for use in link quality indication (LQI). There is therefore no added complexity in reusing existing SNR estimates to select an appropriate MMSE channel estimation filter.

Coherence bandwidth is an effective measure of subcarrier correlation and is inversely proportional to the channel root mean square (RMS) delay spread [10]. Although this makes RMS delay-spread an excellent classifier, it is not practical given that it can only be calculated after the channel estimate has been made.

We therefore propose a much coarser metric of coherence bandwidth that we define as the zero-crossing rate of the ZF channel estimate and denote as $\hat{\tau}$. $\hat{\tau}$ is easily calculated by adding the exclusive-OR of the sign-bit of each tap in the ZF channel estimate. For example, in an MB-OFDM system, this will produce an adder-tree with $\log _{2} N=8$ levels. Since the inputs to this tree are only 1-bit wide, the final output will be 8-bits if full adders are used. We therefore conclude that calculating $\hat{\tau}$ does not add significant incremental complexity.

Having thus defined low-complexity quantitative estimates for both SNR and subcarrier correlation, we now consider the calculation of $S \times K$ MMSE channel estimation filters via Alg. 2. As each of the $S \times K$ MMSE filters require a non-linear optimization, this algorithm is computationally expensive and can only be performed off-line.

By classifying CIR by SNR and subcarrier correlation, we can reduce MSE by matching the MMSE channel estimation filter to instantaneous channel conditions. Although both the SNR estimates and subcarrier correlation estimates are corrupted by AWGN, we note that the worst-case impact of poor classification is no improvement over no classification. For example, consider the case where $\hat{\tau}$ is totally corrupted and contains no useful information. The resultant categorization of CIR will be entirely random. The $K$ independent $\mathbf{R}_{h h}$ will therefore be equivalent. Now consider the case where $\hat{\tau}$ is only roughly proportional to channel delay spread. The 'long' and 'short' channels will be grouped together and this will cause

\footnotetext{
${ }^{2}$ Ideally, the $K$ channel categories will be defined such that there is a $\frac{1}{K}$ probability of a given CIR being assigned to a given category. This means that each of the $K \mathbf{R}_{h h}$ will be calculated using the same number of channel realizations.
}
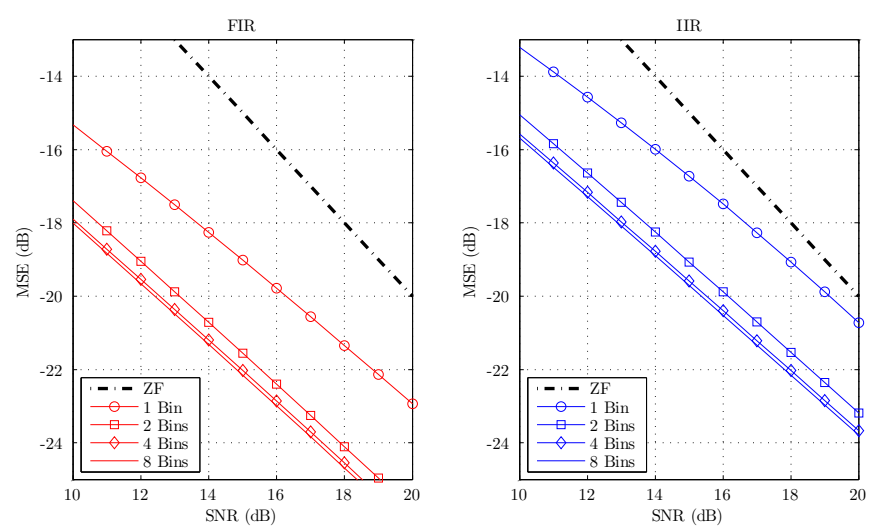

Fig. 4. MSE for adaptive filtering.
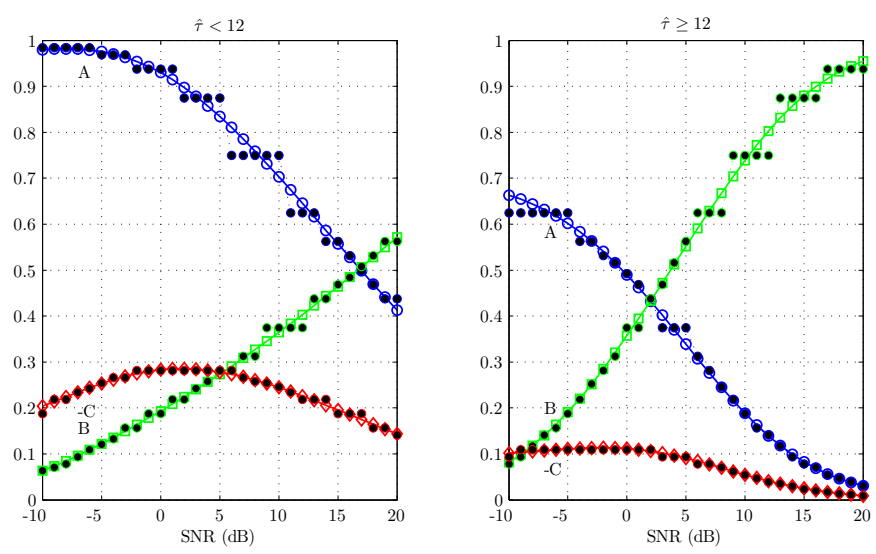

Fig. 5. $\{A, B, C\}$ for a $\mathbf{b}^{\leftrightarrow}$ filter. A $Q=2$ quantization is depicted as $\bullet$.

each category's $\mathbf{R}_{h h}$ to be unique.

Fig. 4 shows how much the MSE is reduced when adaptive filtering is used. In this simulation, the are $S=10$ SNR categories and variable $K=\{1,2,4,8\}$ subcarrier correlation categories. CIRs were obtained randomly from CM1 through CM4. We observe that there is negligible different in performance when $K \geq 4$. Given that the low-order IIR filters can be stored with very few bits of ROM, the gains of adaptive filtering can be realized at little cost.

Fig. 5 shows the coefficients for a set of $\mathbf{b}_{Q=2}^{\leftrightarrow}$ IIR MMSE channel estimation filters when $K=2$ and $S=30$. These filter coefficients were obtained using Alg. 2 and are similar to the $K=4$ filters used in the PER simulations of Section $V$. It can be observed that the filter coefficients for the 'short' channel are different from those for the 'long' channel. The these two types of channel are delineated about $\hat{\tau}=12$, which is set so that there is a $50 \%$ likelihood that a CIR randomly selected from CM1 through CM4 will fall in each category. The figure also shows the quantized PPP coefficients for $Q=2$. It can be seen that the error due to even this highly aggressive quantization is small.

The algorithm of Alg. 1 shows that it is possible to implement adaptive MMSE IIR channel estimation filters at nominal complexity. Each of the $K \times S$ filter realizations shown in Fig. 

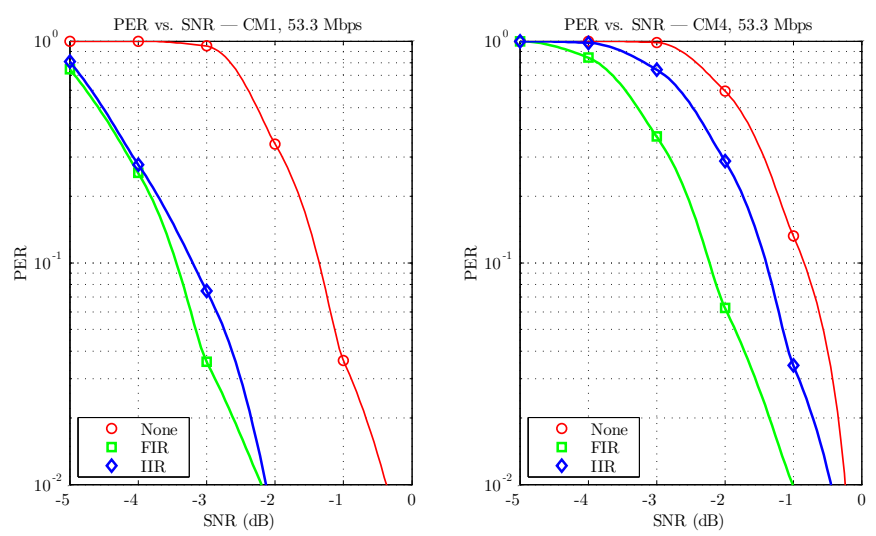

Fig. 6. PER for ZF and adaptive MMSE channel estimation at $53.3 \mathrm{Mbps}$.

5 can be stored in as little as 18 bits of ROM. When $K=2$ and $S=30$, this means that the an adaptive filter library requires as little as 128 bytes of ROM.

\section{RESUlts}

A Monte-Carlo simulation was used to quantify the impact of different approaches to MMSE channel estimation on the PER of an MB-OFDM receiver. The simulation environment implemented the complete MB-OFDM PHY [4] and considers forward error correction (FEC), time-frequency interleaving (TFI), time-domain spreading (TDS), frequency-domain spreading (FDS) and dual-carrier modulation (DCM). Note that no decision-feedback equalization (DFE) is used, which means that the channel estimate is based solely on the channel estimation sequence in the packet preamble. The IIR filters are adaptive to SNR, with $S=30$, and channel length, with $K=4$. The adaptive filters were derived using Alg. 2 and implemented using Alg. 1.

The results of Fig. 6 and Fig. 7 show that there is very little PER difference between optimal FIR channel smoothing and a $\mathbf{b} \overleftrightarrow{\leftrightarrow}=2$ IIR approximation. In most cases, the performance of IIR estimation smoothing is indistinguishable from that of the much higher complexity FIR estimation smoothing. The only time that FIR estimation smoothing is noticeably superior is in a highly frequency-selective CM4 channel at low SNR. Relative to ZF OFDM estimation, we conclude that IIR estimation smoothing offers significant PER improvement at nominal complexity in all channels.
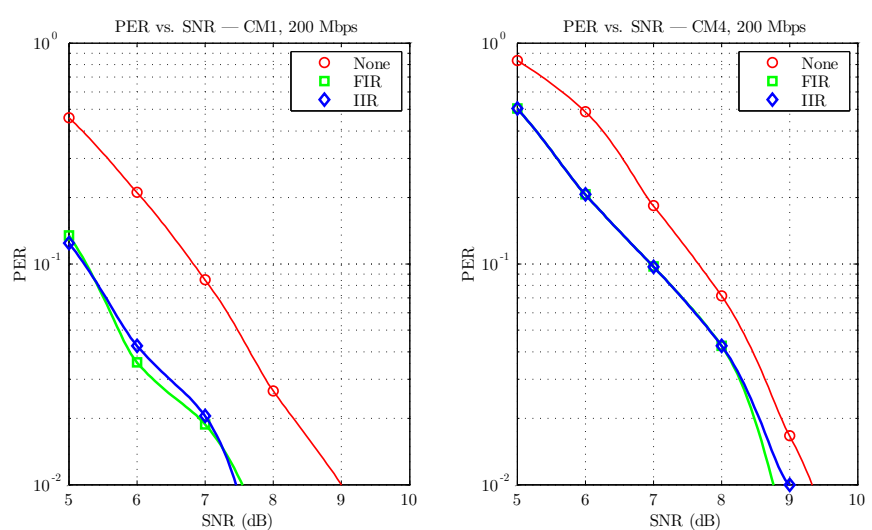

Fig. 7. PER for ZF and adaptive MMSE channel estimation at $200 \mathrm{Mbps}$.

\section{CONCLUSION}

In this paper, we derived an extremely low-complexity IIR approximation to MMSE channel estimation. In the context of MB-OFDM systems, we showed how an IIR filter can be used to achieve up to a $1.5 \mathrm{~dB}$ improvement in PER performance at a cost of less than 46 additions per subcarrier.

\section{REFERENCES}

[1] Z. Wang and G. B. Giannakis, "Wireless multicarrier communications," IEEE Signal Processing Magazine, vol. 17, no. 3, pp. 29-48, 2000.

[2] A. Chini, "Multicarrier modulation in frequency selective fading channels," Ph.D. dissertation, Carlton University Canada, 1994.

[3] A. Batra, J. Balakrishnan, G. R. Aiello, J. R. Foerster, and A. Dabak, "Design of a multiband OFDM system for realistic UWB channel environments," in IEEE Transactions on Microwave Theory and Techniques, vol. 52, no. 9, Sept. 2004, pp. 2123-2138.

[4] High Rate Ultra Wideband PHY and MAC Standard, ECMA International ECMA-368, Dec. 2005.

[5] J. J. van de Beek, O. Edfors, M. Sandell, S. K. Wilson, and P. O. Brjesson, "On channel estimation in OFDM systems," in Proceedings of the IEEE Vehicular Technology Conference, July 1995, pp. 815-819.

[6] R. M. Gray, Toeplitz and Circulant Matrices: A Review. Stanford University, 2006

[7] J. C. Lagarias, J. A. Reeds, M. H. Wright, and P. E. Wright, "Convergence properties of the nelder-mead simplex method in low dimensions," SIAM Journal of Optimization, vol. 9, no. 1, pp. 112-147, 1998.

[8] "DSP implementation techniques for Xilinx FPGAs," Xilinx, Inc., Tech. Rep., 2005.

[9] H.-Y. Liu, Y.-H. Yu, C.-J. Hung, T.-Y. Hsu, and C.-Y. Lee, “Combining adaptive smoothing and decision-directed channel estimation for OFDM WLAN systems," in ISCAS (2), 2003, pp. 149-152.

[10] J. G. Proakis, Digital Communications, 3rd ed. New York: McGrawHill Book Company, 1995. 